\title{
Identification of Bio-oil Compound Utilizing Yeasts Through Phenotypic Microarray Screening
}

\author{
Emily T. Kostas ${ }^{1,2}$ (1) $\cdot$ Mick Cooper $^{3} \cdot$ Benjamin J. Shepherd $^{1} \cdot$ John P. Robinson ${ }^{1}$
}

Received: 18 July 2018 / Accepted: 21 February 2019 / Published online: 26 February 2019

(c) The Author(s) 2019

\begin{abstract}
Biomass pyrolysis bio-oil contains a plethora of carbon sources with the potential to be utilized by microorganisms and converted into high value products. However, the majority of these compounds are either highly toxic to microorganisms or are not directly utilizable. Hence research is required to develop methods of separating microbe friendly compounds from inhibitory ones, and to also identify novel microorganisms that can utilise the principal carbon sources in pyrolysis bio-oil. This study employed a phenotypic microarray (PM) technique that measured yeast metabolic output to screen for and shortlist yeast strains able to metabolize various bio-oil carbon sources, with a focus on the anhydrosugar levoglucosan. Four strains of yeast (two Pichia spp. and two Kluyveromyces spp.) were shortlisted due to their high metabolic output (between 79.7 and 113.7 redox signal intensity) on levoglucosan from the PM assay. Under anaerobic fermentation conditions the strains were able to uptake levoglucosan (between 79 and 100\% uptake efficiency) but not produce bioethanol; yet trace amounts of acetic acid were generated. This study demonstrated the application of applying the PM technique to screen for novel yeast strains with abilities to metabolize compounds present in pyrolysis bio-oil that could lead to the identification of novel levoglucosan utilization pathways.
\end{abstract}

\section{Graphical Abstract}

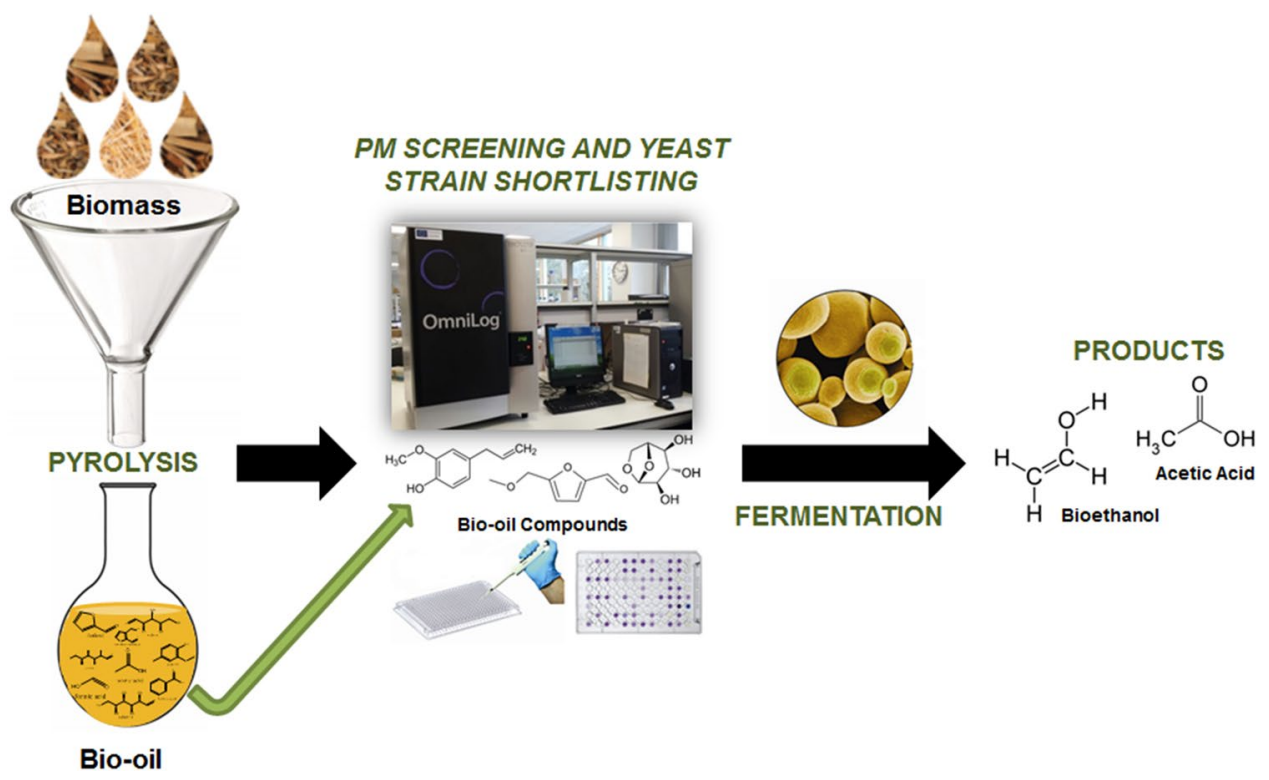

Keywords Phenotypic microarray $\cdot$ Yeast $\cdot$ Pyrolysis $\cdot$ Bio-oil compound $\cdot$ Fermentation

Extended author information available on the last page of the article 


\section{Statement of Novelty}

The need to identify novel yeast strains with abilities to utilise pyrolysis bio-oil is of increasing significance for downstream bio-processing into higher value products. However, traditional methods of yeast screening is time consuming and laborious. In this study, a phenotypic microarray technique was successfully used for the first time as a high-throughput screening tool for identification of novel yeast strains with abilities to metabolize pyrolysis bio-oil carbon sources. Shortlisted strains were confirmed by growth tests and then laboratory-scale anaerobic fermentations for carbon end-point production, of which acetic acid was identified. This work will impact future developments in microbial-conversion processes of biooil, since there is a need to identify candidate genes in microorganisms which are responsible for unique bio-oil compound uptake.

\section{Introduction}

In order for a future bio-based economy to be viable, efficient methods of converting renewable feedstocks into high value bio-based products need to be established and optimised. Furthermore for the biotechnological conversion of such feedstocks, it is necessary to utilise processing technologies capable of yielding monomers or building blocks that are suitable for microbial metabolism [1]. Thermo-chemical biomass processes such as pyrolysis has become an expanding area of research in recent years due to its economical advantages. Pyrolysis is defined as the thermal decomposition of biomass in the absence of oxygen, resulting in the formation of three main products; bio-char, liquid bio-oil and bio-gas (syngas) [2]. Bio-oil (or pyrolysis-oil/pyrolysate) is an easily storable and transportable energy rich liquid medium elementally resembling the source of biomass; mainly water (15-30 wt \%) and more than 100 different organic compounds [3] including furans, aldehydes, phenols, acids and anhydrosugars. Its rich chemical composition makes it not only a viable source for the thermo-chemical-based bio-refinery which enables the production of platform chemicals and conventional biofuels [4], but also as a direct fermentation substrate. This concept of combining thermal biomass deconstruction followed by subsequent biological processing has been termed 'hybrid processing' [5-7]. Polysaccharide derived substrates such as the anhydrosugar 'levoglucosan' is the most abundant sugar in bio-oil; making it a direct target for microbial processing. Nonetheless, aromatic compounds (e.g. phenol, guaiacol, syringol) and heterocyclic compounds (e.g. furfural) present in the complex medium makes bio-oil inhibitory to microorganisms and most lack the ability to directly metabolize levoglucosan [8].

Several studies have successfully converted levoglucosan to glucose by acid hydrolysis [9-13] followed by the subsequent fermentation of glucose to ethanol via different microorganisms. However, the inclusion of a hydrolysis step to produce fermentable glucose from levoglucosan is not only costly, but may also result in the formation of additional inhibitory compounds [14]. In order to overcome the potential bottlenecks that could hinder the use of bio-oil as a direct fermentation substrate and to enhance future prospects for a more consolidated biological process that produces fuels or chemicals directly from bio-oil, research is required to underpin (i) the separation of microbe-friendly compounds, such as levoglucosan, from the complex bio-oil, and (ii) the screening and identification of microorganisms that are capable of utilizing and (iii) converting such compounds into high value products.

The search for new microorganisms with abilities to use levoglucosan as a carbon source has become an increasing area of research. It has been found that many eukaryotic and prokaryotic microorganisms can indeed directly metabolize levoglucosan through their levoglucosan utilization pathways [15-17]. In the specific case for eukaryotes, which have a much more developed and advanced levoglucosan utilization pathway than prokaryotes [17], organisms such as filamentous fungi and several yeast strains isolated from soil contain a levoglucosan kinase which directly converts levoglucosan into glucose-6-phosphate $[18,19]$ and routes it into the general glycolytic pathway. Despite the fact that several fungi have been shown to convert levoglucosan into itaconic acid [16] and citric acid [18], most other natural levoglucosan utilizers are not able to produce ethanol, and even if some can produce ethanol, the yields are extremely low [20]. Therefore, scientists are engineering strains by importing the levoglucosan pathway found in natural strains of fungi and yeast into organisms such as Escherichia coli [21,22], Rhodococcus spp. [23] and Pseudomonas spp. [24] for ethanol and lipid production. However, searching for new organisms with high activities for the conversion of levoglucosan into glucose-6-phosphate and its subsequent utilization is a very laborious and time consuming task.

The application of a novel phenotypic microarray (PM) has proved to be an efficient, accurate and rapid screening tool to shortlist candidate yeast strains across a number of different biotechnological applications. The PM approach has previously been demonstrated to be successful in identifying strains for efficient second generation bioethanol production [25, 26], to measure tolerance to stresses on Saccharomyces spp. yeast during fermentation [27], to determine the metabolic profiling of yeast on different pre-treatment 
hydrolysates [28] and to identify yeast strains with abilities to metabolise monosaccharides derived from macroalgae [29]. The technique measures the metabolic activity of yeast cells against various media (containing carbon sources of interest), therefore serving as a high-throughput screening tool to shortlist candidate yeast strains.

The aim of this work was to identify novel yeast strains that can utilise levoglucosan directly using a PM screening approach. Particularly with pyrolysis technologies that have adopted microwave heating to drive the pyrolysis process, higher yields of levoglucosan have been quantified in the generated bio-oil compared to conventionally heated pyrolysis $[30,31]$; highlighting the attractiveness of this technology to produce sufficient amounts of this anhydrosugar for subsequent downstream processing. Nonetheless, the process still produces pyrolysis bio-oil containing thermal degradation compounds, such as 5-HMF, furfural and phenol that are detrimental to the growth of yeast. As previous studies have already PM screened for yeast strain tolerance towards the aforementioned growth inhibiting compounds [27], other compounds that are also identifiable in pyrolysis bio-oil were incorporated into this study to enhance its scope (including benzoic acid, $p$-coumaric acid, levulinic acid and syringic acid).

The strains used in this study (mainly Saccharomyces, Pichia, Candida and Kluyveromyces) were selected based upon the fact that they had not been previously investigated for levoglucosan or even bio-oil compound utilisation. Yeast strains which displayed high metabolic activity when cultured on media containing a single bio-oil compound of interest were shortlisted and further screened for confirmation of growth, and finally selected yeast strains were examined in laboratory-scaled anaerobic fermentations of the bio-oil compounds for possible carbon-end point production. To the authors' knowledge, no studies have been published using the PM for screening of yeast strains on the pyrolysis bio-oil derived compounds investigated in this study.

\section{Materials and Methods}

\section{Chemicals and Reagents}

Levoglucosan, benzoic acid, levulinic acid, syringic acid and $p$-coumaric acid were all of analytical grade and were supplied by Sigma (Dorset, UK). Other chemicals were standard laboratory reagents.

\section{Yeast Strains and Growth Conditions}

All yeast strains that were investigated in this study (listed in Table 1) were taken from $-80^{\circ} \mathrm{C}$ glycerol stocks and inoculated into a liquid medium containing $10 \mathrm{~g} / \mathrm{L}$ yeast extract, $20 \mathrm{~g} / \mathrm{L}$ peptone and $20 \mathrm{~g} / \mathrm{L}$ glucose (YPD). Yeasts were then placed into an orbital shaker (180 rpm; CERTOMAT BS-1 incubator, Germany) at $30^{\circ} \mathrm{C}$ for $48 \mathrm{~h}$. One hundred microliters of these cells were then spread onto YPD slopes and incubated at $30^{\circ} \mathrm{C}$ for a further $48 \mathrm{~h}$.

\section{Phenotypic Microarray (PM) Analysis (Biolog-Omnilog)}

The Bio-log Omnilog (Biolog, Hayward, CA, USA) was used as screening tool to measure the metabolic activity of yeast when cultured on minimal synthetic media containing compounds originally derived from bio-oil. Synthetic minimal media was prepared using $0.67 \%(w / v)$ yeast nitrogen

Table 1 List of yeast strains used in this study

\begin{tabular}{llll}
\hline Microorganism & Source & Strain ID & Additional strain information $^{\text {a }}$ \\
\hline Saccharomyces cerevisiae & NCYC & NCYC 2592 & Distillers production strain \\
Candida succiphila & NCYC & NCYC 1403 & Isolated from sap of Amygdalus persica (peach tree), Japan \\
Candida arabinofermentans & NCYC & NCYC 2916 & Isolated from insect frass from dead Larix sp. (larch tree), Fairbanks, Alaska, USA \\
Pichia guilliermondii & NCYC & NCYC 443 & Isolated from insect frass from Ulmus Americana (elm tree), Peoria, Illinois, USA \\
Pichia etchellsii & NCYC & NCYC 740 & Isolated from fermenting cucumbers, in 15\% brine with 0.61\% lactic acid \\
Pichia membranifaciens & NCYC & NCYC 101 & Isolated from Kefir beer \\
Pichia angusta & NCYC & NCYC 1457 & Isolated from soil irrigated with distillery effluent, Pernambuco, Brazil \\
Kluyveromyces dobzhanskii & NCYC & NCYC 538 & Isolated from Drosophila pseudoobscura (fruit fly), Piñon Flat, California, USA \\
Kluyveromyces wickerhamii & NCYC & NCYC 546 & Isolated from Drosophila sp. (fruit fly), California, USA \\
Kluyveromyces africanus & NCYC & NCYC 2729 & Isolated from soil, Transvaal, South Africa \\
Kluyveromyces lodderae & NCYC & NCYC 1417 & Isolated from soil, South Africa \\
Saccharomyces uvarum & BSYC & S. uvarum & N.A \\
Saccharomyces eubayanus & BSYC & S. eubayanus & N.A \\
\hline
\end{tabular}

NCYC National Collection of Yeast Cultures, BSYC Brewing Science Yeast Cultures

${ }^{a}$ Information from NCYC website (http://www.ncyc.co.uk) 
base (YNB) and $0.2 \mu \mathrm{L}$ of tetrazolium dye $\mathrm{D}$ (Biolog, Hayward, CA, USA). The $\mathrm{pH}$ was within the range of 5.2-5.6 at a $0.67 \%(\mathrm{w} / \mathrm{v})$ solution. Glucose (which served as a positive control) and levoglucosan (at $3 \% \mathrm{w} / \mathrm{v}$ ) and $p$-coumaric acid and levulinic acid (at $0.5 \% \mathrm{w} / \mathrm{v}$ ) were individually supplemented before final volumes were made up to $60 \mu \mathrm{L}$ using RO sterile water and aliquoted into individual wells of the PM plates. For experiments investigating the metabolic activity of yeast on benzoic acid, $p$-coumaric acid and syringic acid, were set up as above, however stock solutions were prepared using $100 \%$ methanol (20\% stocks benzoic acid and $p$-coumaric acid; $10 \%$ stock syringic acid). The amount of RO sterile water added was modified to make up a final concentration of $0.5 \%$ of each of the three above. Yeast strains $(60 \mu \mathrm{L})$ were prepared for inoculation into the PM assay plates according to the method outlined in Kostas et al. [29]. Wells containing only synthetic minimal media containing individual carbon sources without yeast cells were also included in the PM plates to serve as negative controls. Prepared plates were vacuum sealed as described in Kostas et al. [29] and were then placed into the Omnilog reader at $30{ }^{\circ} \mathrm{C}$ for $95 \mathrm{~h}$. The Omnilog reader was programmed to photograph the PM plates every $15 \mathrm{~min}$, converting the pixel density of each well into a signal data value that represents cell metabolic output and dye colour change. The reduction of the dye, producing a colour change from colourless to violet, results from the cells' metabolic activity when in contact with the different media in each well; this has been defined here as redox signal intensity. The redox signal intensity data was assembled in the Biolog software and exported using Microsoft Excel. All assays were conducted in triplicate and the mean values are presented.

\section{Confirmation of PM Results Measurement of Yeast Growth}

Positive combinations of yeast strains and individual bio-oil carbon sources were tested under identical growth conditions as for the PM assays described in the previous section. PM plates were incubated at $30{ }^{\circ} \mathrm{C}$ for $95 \mathrm{~h}$ under a nitrogen flow in order make conditions anaerobic, with a reading taken every $15 \mathrm{~min}$ using a TECAN (Mannedorf, Switzerland) Infinite M200 Pro plate reader. Assays were performed in triplicate.

\section{Fermentations of bio-oil compounds using selected yeast strains}

Yeast strains which were metabolically more active compared to their corresponding glucose redox signal intensities (RSI) on the bio-oil compounds and were subsequently confirmed by growth tests were directly used for fermentation trials. Fermentation vessels (30 mL; Wheaton, USA) containing $25 \mathrm{~mL}$ of media constituting of $2 \%$ peptone, $1 \%$ yeast extract and either 3\% levoglucosan/ glucose (positive control) or $0.5 \%$-coumaric acid/levulinic acid were inoculated with the selected yeast strain at a pitching rate of ca. $10^{6}$ cells $/ \mathrm{mL}$. Anaerobic conditions were prepared using a sealed butyl bung (Fischer, Loughborough, UK) and aluminium caps (Fischer Scientific). A hypodermic needle attached with a Bunsen valve was purged through the rubber septum to facilitate the release of any $\mathrm{CO}_{2}$. The vessels were incubated at $30{ }^{\circ} \mathrm{C}$ (MIR-253 incubator, Sanyo Electric Co., Japan) with magnetic stirring set at $120 \mathrm{rpm}$. Samples were taken at the end of the fermentation for carbon source utilization and carbon end point production analysis via HPLC. All fermentations were carried out in triplicate.

\section{High Performance Liquid Chromatography (HPLC) and Gas Chromatography (GC)}

Levoglucosan was quantified by HPLC using an AS-2055 Intelligent Auto-sampler and a PU-1580 Intelligent HPLC Pump (Jasco, Japan). The Rezex ROA Organic Acid $\mathrm{H}+$ organic acid column $(5 \mu \mathrm{m}, 7.8 \mathrm{~mm} \times 300 \mathrm{~mm}$; Phenomenex, UK) was operated at ambient temperature with a mobile phase of $0.005 \mathrm{~N} \mathrm{H}_{2} \mathrm{SO}_{4}$, at a flow rate of $0.5 \mathrm{~mL} /$ min. A Refractive Index cell (RI-2031 Intelligent Refractive Index detector, Jasco, Japan) was used for detection, and the injection volume was $10 \mu \mathrm{L}$. Data were acquired using the Azur software package (v. 4.6.0.0; Datalys, France). Prior to HPLC analysis, all samples and standards were filtered using Whatman GD/X syringe filters (GF/C $25 \mathrm{~mm}$ filter diameter/1.2 $\mu \mathrm{m}$ pore size; Whatman, UK). All analyses were conducted in triplicate.

The analysis of $p$-coumaric acid was quantified by HPLC using UV detection at $270 \mathrm{~nm}$ (2695 HPLC system and 996 Photodiode Array Detector, Waters, USA) and a Techsphere ODS C18 column $(5 \mu \mathrm{m}, 4.6 \mathrm{~mm} \times 250 \mathrm{~mm}$; HPLC Technologies, UK) was used at room temperature. The sample volume was $10 \mu \mathrm{L}$, and the mobile phase was a gradient of methanol in $1 \%$ acetic acid at an overall flow rate of $1.0 \mathrm{~mL} \mathrm{~min}^{-1}$. The methanol concentration was increased from 20 to $50 \%$ over 30 min with a $100 \%$ methanol column cleaning phase and a 9-min re-equilibration period. Data were recorded using Millennium Chromatography software (Waters, USA).

For the identification of fermentation carbon end point products gas chromatography-high resolution mass spectrometry (GC-HRMS) was carried out, using a JEOL GCX GC-ToFMS (time of flight mass spectrometry) (JEOL Corp. Tokyo, Japan). Analysis conditions were as follows. The GC temperature program was: $40{ }^{\circ} \mathrm{C}$ (hold $3 \mathrm{~min}$ ), then increased to $260{ }^{\circ} \mathrm{C}$ at $5{ }^{\circ} \mathrm{C} / \mathrm{min}$. The GC column installed was a Thermo Scientific TraceGold TG-POLAR $(30 \mathrm{~m} \times 0.25 \mathrm{~mm} \times 0.25 \mu \mathrm{m}$; ThermoFisher Scientific, 
Waltham, USA). Sample aliquots of $1 \mu \mathrm{L}$ were injected in splitless mode at $220^{\circ} \mathrm{C}$. Helium was employed as the carrier gas, with a flow rate $1 \mathrm{~mL} / \mathrm{min}$. The mass spectrometer was operated in EI mode (70 eV ionisation energy) with a detection range of 50-600 Da. The ion source temperature was held at $250{ }^{\circ} \mathrm{C}$. Peaks that had a high degree of certainty (over $85 \%$ ) were reported. Acetic acid was observed as a minor component in the total ion chromatogram (TIC) data, but was more readily noted when extracted ion chromatograms (EIC) were constructed from the mass spectral signal acquired over the mass range 60.01-60.04 Da. In all samples, the centroided mass of the molecular ion of acetic acid was determined as $60.025 \pm 0.001 \mathrm{Da}$. In addition, the GC retention index (RI) of the proposed acetic acid chromatographic peak agreed with that reported in the NIST14 mass spectral library (Polar GC column: 1449 \pm 13 (380); National Institute of Standards and Technology, Gaithersburg, USA).

\section{Results}

\section{Phenotypic Microarray Analysis of Yeast Strains}

The redox signal intensity values (which are indicative of cell metabolic activity) of yeast strains investigated in this study on synthetic minimal media containing an individual bio-oil compound can be seen in Fig. 1a-f. Glucose has been included to serve as a positive control and as a benchmark to compare to the RSI values of the bio-oil compounds. As expected, there were measureable metabolic outputs for all yeast strains on glucose (Fig. 1a) after $96 \mathrm{~h}$ of incubation at $30{ }^{\circ} \mathrm{C}$; between 34 and 100 RSI. The yeast strain Saccharomyces cerevisiae NCYC 2592 gave the lowest RSI value of 34, despite previous studies having shown that RSI levels of this strain on glucose can reach between 75 and 100 RSI [26, 29]. Reasons for its relatively low metabolism on glucose in this study are not clear however due to its negligible metabolism on the main carbon sources of interest in this study, it was not included in any further experiments. It is evident that there also were measureable metabolic outputs on minimal media containing 3\% (w/v) levoglucosan (Fig. 1d) with yeast strains Pichia etchellsii NCYC 740, Kluyveromyces dobzhanskii NCYC 538 and Kluyveromyces africanus NCYC 2729 displaying values of 73.3, 57.3 and 67.7 RSI, respectively. Interestingly, yeast strains Pichia membranifaciens NCYC 101 and Pichia angusta NCYC 1457 also exhibited relatively high RSI values on $0.5 \%(\mathrm{w} / \mathrm{v})$ p-coumaric acid, with values as high as 75.5 and $65.0 \mathrm{RSI}$, respectively. The majority of the strains however displayed low metabolic outputs on benzoic acid, syringic acid and levulinic acid with the highest RSI values not exceeding above ca. 22.0 (Fig. 1b, e, and f). Strains that displayed RSI levels lower than 20 on any carbon source were not taken forward for further investigation.

Table 2 shows the normalised metabolic activity values (compared to their glucose utilization) of all the yeast strains on the different bio-oil compounds. There appeared to be no direct correlation between glucose RSI values and the RSI values of the yeast strains when metabolizing the bio-oil derived compounds. There were a number of strains however which displayed higher RSI values on certain bio-oil compounds in comparison to glucose. These included strains P. membranifaciens NCYC 101 which had an RSI value of 129.1 on $p$-coumaric acid (58.5 RSI on glucose), and strains K. dobzhanskii NCYC 538 and K. africanus NCYC 2729 which had RSI values of 96.4 and 113.7 on levoglucosan (glucose RSIs of 59.5 for both strains), respectively. The yeast strains mentioned above were subsequently shortlisted for further investigation. Two more yeast strains were also shortlisted for further trials, as RSI values on certain biooil compounds were almost as high as their corresponding glucose values; strains $P$. angusta NCYC 1457 on $p$-coumaric acid and P. membranifaciens NCYC 101 on levoglucosan. The metabolic profile curves of the aforementioned strains that appeared to look promising on their respective carbon sources and glucose (positive control) can be seen in Fig. 2a-f. It is evident that for all strains, the rate of metabolism on their selected carbon source is slower with much longer lag phases than metabolism on glucose, which is expected. Strains on levoglucosan appeared to attenuate much faster (normally after $50 \mathrm{~h}$ ) compared to the strains on $p$-coumaric acid which took up $74 \mathrm{~h}$ to reach attenuation phase. However, strains P. membranifaciens NCYC 101, K. dobzhanskii NCYC 538, K. africanus NCYC 2729 and $P$. etchellsii NCYC740 all appeared to display similar metabolic profiles on levoglucosan albeit at different RSI levels. Interestingly, K. dobzhanskii NCYC 538 was the only strain to exhibit a higher RSI level than its metabolism on glucose from around $50 \mathrm{~h}$ onwards.

\section{Growth Measurements}

Strains that were metabolically active on the bio-oil compounds tested in PM assay were selected for further experiments to confirm their abilities to grow on the same carbon sources (Fig. 3a-e). Growth was confirmed for all shortlisted yeast strains on what appeared to be their compatible bio-oil carbon sources.

\section{Screening for Carbon-End Point Products in Laboratory-Scale Fermentations}

Trial fermentations were performed with yeast strains P. membranifaciens NCYC101, P. etchellsii NCYC740, K. dobzhanskii NCYC538 and K. africanus NCYC 2729 

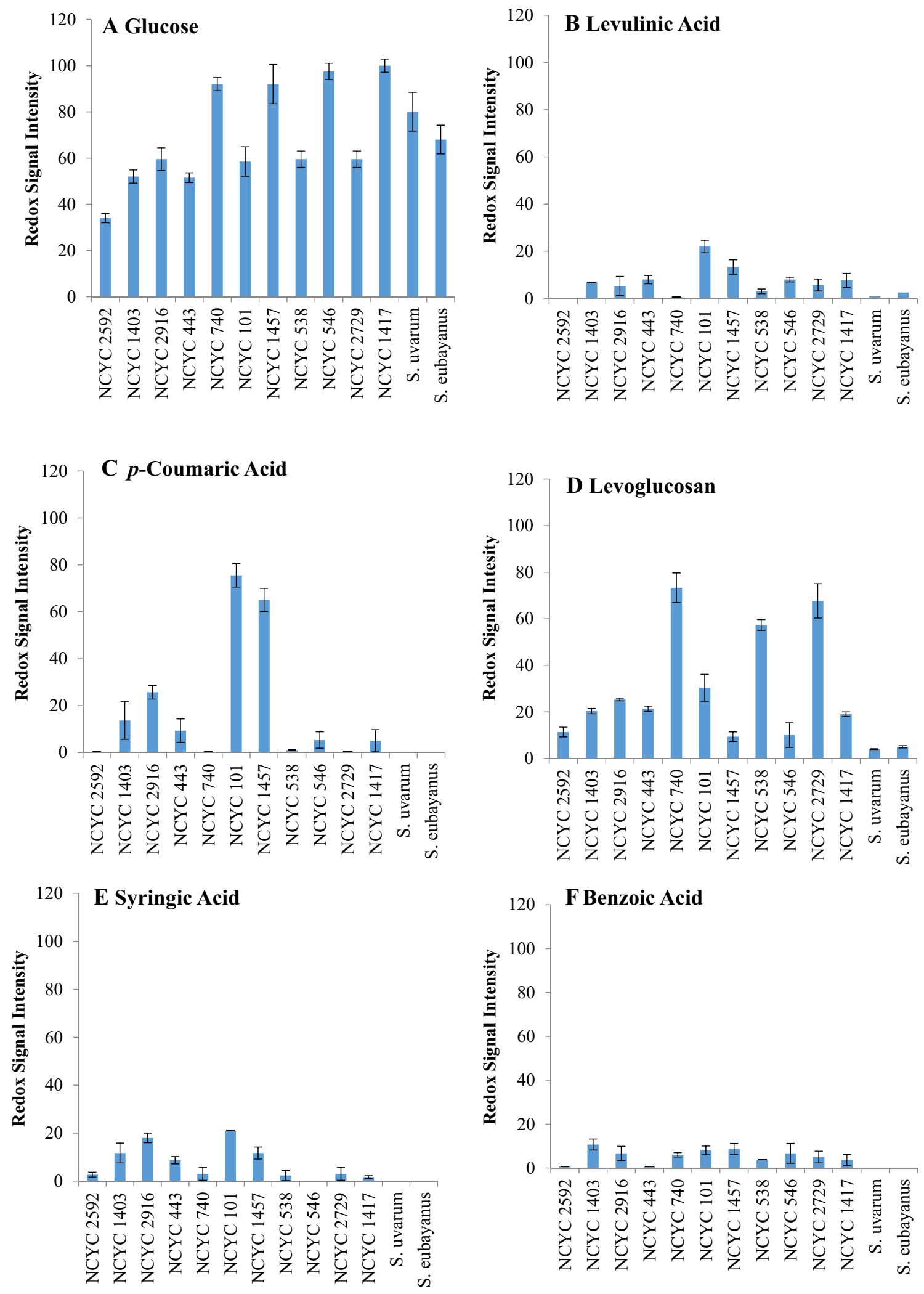

Fig. 1 Phenotypic microarray analysis (redox signal intensity) at $96 \mathrm{~h}$ for yeast strains on minimal synthetic media containing: a 3\% glucose, b $0.5 \%$ levulinic acid, c $0.5 \%$ p-coumaric acid, d $3 \%$ levo-

glucosan, e $0.5 \%$ syringic acid and $\mathbf{f} 0.5 \%$ benzoic acid. Data are the mean \pm SD of three measurements 
Table 2 Phenotypic microarray results (RSI at 96 h) for each yeast on bio-oil compounds investigated in this study relative to their corresponding glucose values (RSI)

\begin{tabular}{lcccccc}
\hline Yeast & Glucose (RSI) & \multicolumn{4}{c}{ Bio-oil compound (\% RSI in comparison to glucose) } \\
\cline { 2 - 6 } & & Levulinic acid & p-Coumaric acid & Levoglucosan & Syringic acid & Benzoic acid \\
\hline NCYC 2592 & $34.0 \pm 2.0$ & $0.0 \pm 0.0$ & $1.0 \pm 0.0$ & $33.3 \pm 2.5$ & $0.0 \pm 0.0$ & $2.0 \pm 0.0$ \\
NCYC 1403 & $52.0 \pm 2.8$ & $13.5 \pm 1.7$ & $26.3 \pm 8.0$ & $39.1 \pm 2.9$ & $0.0 \pm 0.0$ & $20.5 \pm 1.4$ \\
NCYC 2916 & $59.5 \pm 4.9$ & $9.0 \pm 0.6$ & $43.1 \pm 2.9$ & $42.6 \pm 3.0$ & $0.0 \pm 0.0$ & $11.2 \pm 0.5$ \\
NCYC 443 & $51.5 \pm 2.1$ & $15.5 \pm 2.8$ & $18.1 \pm 5.0$ & $41.4 \pm 4.1$ & $0.0 \pm 0.0$ & $1.3 \pm 0.2$ \\
NCYC 740 & $92.0 \pm 2.8$ & $0.7 \pm 0.1$ & $0.4 \pm 0.0$ & $79.7 \pm 5.7$ & $0.0 \pm 0.0$ & $6.5 \pm 2.0$ \\
NCYC 101 & $58.5 \pm 6.4$ & $37.6 \pm 3.1$ & $129.1 \pm 5.0$ & $51.9 \pm 3.1$ & $0.0 \pm 0.0$ & $13.7 \pm 3.1$ \\
NCYC 1457 & $92.0 \pm 8.5$ & $14.5 \pm 1.1$ & $70.7 \pm 5.0$ & $10.1 \pm 0.9$ & $0.0 \pm 0.0$ & $9.4 \pm 2.9$ \\
NCYC 538 & $59.5 \pm 3.5$ & $5.0 \pm 0.8$ & $1.7 \pm 0.0$ & $96.4 \pm 2.9$ & $0.0 \pm 0.0$ & $6.2 \pm 1.1$ \\
NCYC 546 & $97.5 \pm 3.5$ & $8.2 \pm 1.2$ & $5.5 \pm 1.5$ & $10.3 \pm 1.2$ & $0.0 \pm 0.0$ & $6.8 \pm 1.6$ \\
NCYC 2729 & $59.5 \pm 3.5$ & $9.5 \pm 2.0$ & $1.1 \pm 0.0$ & $113.7 \pm 3.8$ & $0.0 \pm 0.0$ & $8.4 \pm 2.1$ \\
NCYC 1417 & $100.0 \pm 2.8$ & $7.7 \pm 0.9$ & $5.0 \pm 1.7$ & $19.0 \pm 1.6$ & $0.0 \pm 0.0$ & $3.7 \pm 0.4$ \\
Saccharomyces uvarum & $80.0 \pm 8.4$ & $1.1 \pm 0.8$ & $0.0 \pm 0.0$ & $5.0 \pm 1.1$ & $0.0 \pm 0.0$ & $0.0 \pm 0.0$ \\
Saccharomyces eubayanus & $68.0 \pm 6.2$ & $3.7 \pm 1.2$ & $0.0 \pm 0.0$ & $7.4 \pm 0.9$ & $0.0 \pm 0.0$ & $0.0 \pm 0.0$ \\
\hline
\end{tabular}

Data are the mean \pm SD of three measurements

in order to identify whether they were able to anaerobically ferment the bio-oil compounds they exhibited a high metabolic activity on from the PM screening assay. It appeared that after 6 days of fermenting a YPD fermentation medium containing $3 \%(\mathrm{w} / \mathrm{v})$ levoglucosan at $30{ }^{\circ} \mathrm{C}$, yeast strains $P$. membranifaciens NCYC 101, P. etchellsii NCYC 740 and K. dobzhanskii NCYC 538 were able to uptake all of the levoglucosan present in the fermentation medium, however $0.63 \% \pm 0.08$ levoglucosan remained at the end of the fermentation with $K$. africanus NCYC2729 (Table 3). Surprisingly K. africanus NCYC 2729 was not able uptake $p$-coumaric acid under anaerobic fermentation conditions, despite the high RSI values exhibited on the PM assay.

Acetic acid was the only identifiable carbon end point product that was produced from the fermentations containing 3\% levoglucosan by yeast strains P. membranifaciens NCYC 101, P. etchellsii NCYC 740, K. dobzhanskii NCYC 538 and K. africanus NCYC 2729; however levels were extremely low and at trace level (Table 4; Fig. 4a). Extracted ion chromatography (EIC) levels were within the ranges of $5.52 \times 10^{4}$ to $1.51 \times 10^{5}$, with strain $K$. africanus NCYC 2729 displaying the highest EIC value of acetic acid. An example of a chromatogram confirming the EIC trace of acetic acid from the fermentation of levoglucosan by $P$. membranifaciens NCYC101 is depicted in Fig. 4b. The two other peaks that appear to be present in the chromatogram (between 26-28 $\mathrm{min}$ and 32-34 min) were not identified, despite being present in all chromatograms and may represent compounds found in the starting media that was then fermented.

\section{Discussion}

In this study, yeast cells' metabolic activity on pyrolysis oil derived carbon sources were employed as a screening tool to identify yeast strains for bio-oil utilization. Yeast that are able to metabolise the carbon source present in the assay change the colour of the dye from colourless to purple; with the intensity of the purple colour directly corresponding to the degree of metabolic activity and thus generates higher RSI values. However it is not possible to determine the metabolic products (for example bioethanol) that may be produced by the yeast in the assay, only metabolic activity; hence it's application as a high through-put screening tool. In previous studies, growth tests on such compounds have often been the followed methodological approach. Yeast species such as Pichia stipitis [32], Rhodosporidium toruloides and Rhodotorula glutinis [33] and Lipomyces starkeyi [22, 34] have been grown on levoglucosan in order to either be identified or for confirmation that they can assimilate levoglucosan and yeast species such as Candida utilise and Schwanniomyces castelli can in fact partially ferment levoglucosan [35]. Yeast strains $K$. africanus NCYC 2729 and K. dobzhanskii NCYC 538 had the highest RSI values on 3\% (w/v) levoglucosan in comparison to glucose, reaching RSI levels as high as 113.7 and 96.4, respectively (Table 2). Kluyveromyces spp. have previously attracted much attention for their potential in the biotechnology sector. The major common feature of $K$. marxianus and Kluyveromyces lactis is the capacity to assimilate lactose and utilise this sugar as a carbon source; 
Fig. 2 Phenotypic microarray analysis (redox signal intensity) time course for shortlisted yeast on selected carbon source and $3 \%$ glucose (control). a Pichia membranifaciens NCYC 101 on levoglucosan, b Pichia membranifaciens NCYC 101 on $p$-coumaric acid, c Pichia angusta NCYC 1457 on p-coumaric acid, d Kluyveromyces africanus NCYC 2729 on levoglucosan, e Kluyveromyces dobzhanskii NCYC 538 on levoglucosan, and $\mathbf{f}$ Pichia etchellsii NCYC 740 on levoglucosan. Data are the mean \pm SD of three measurements
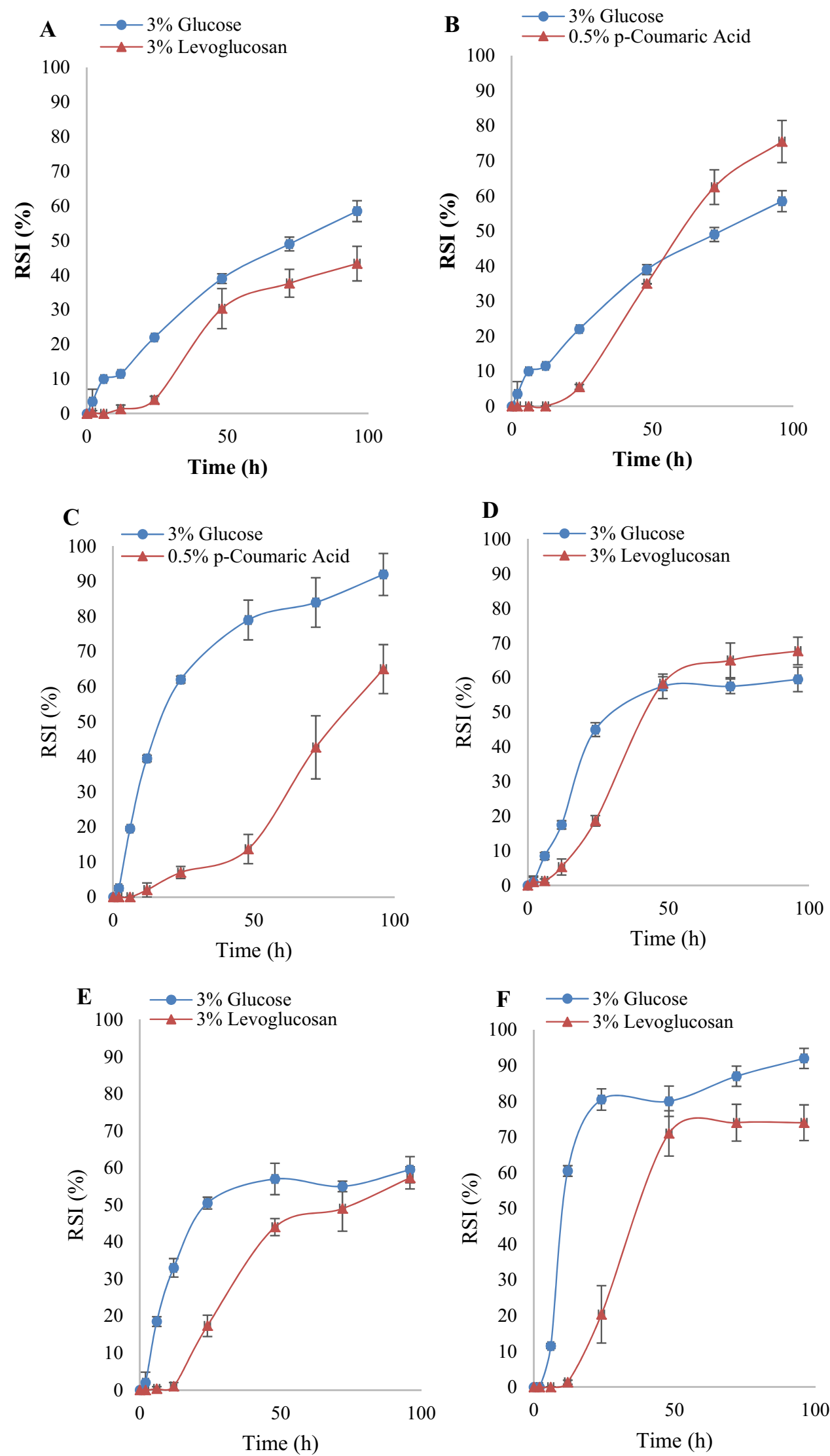

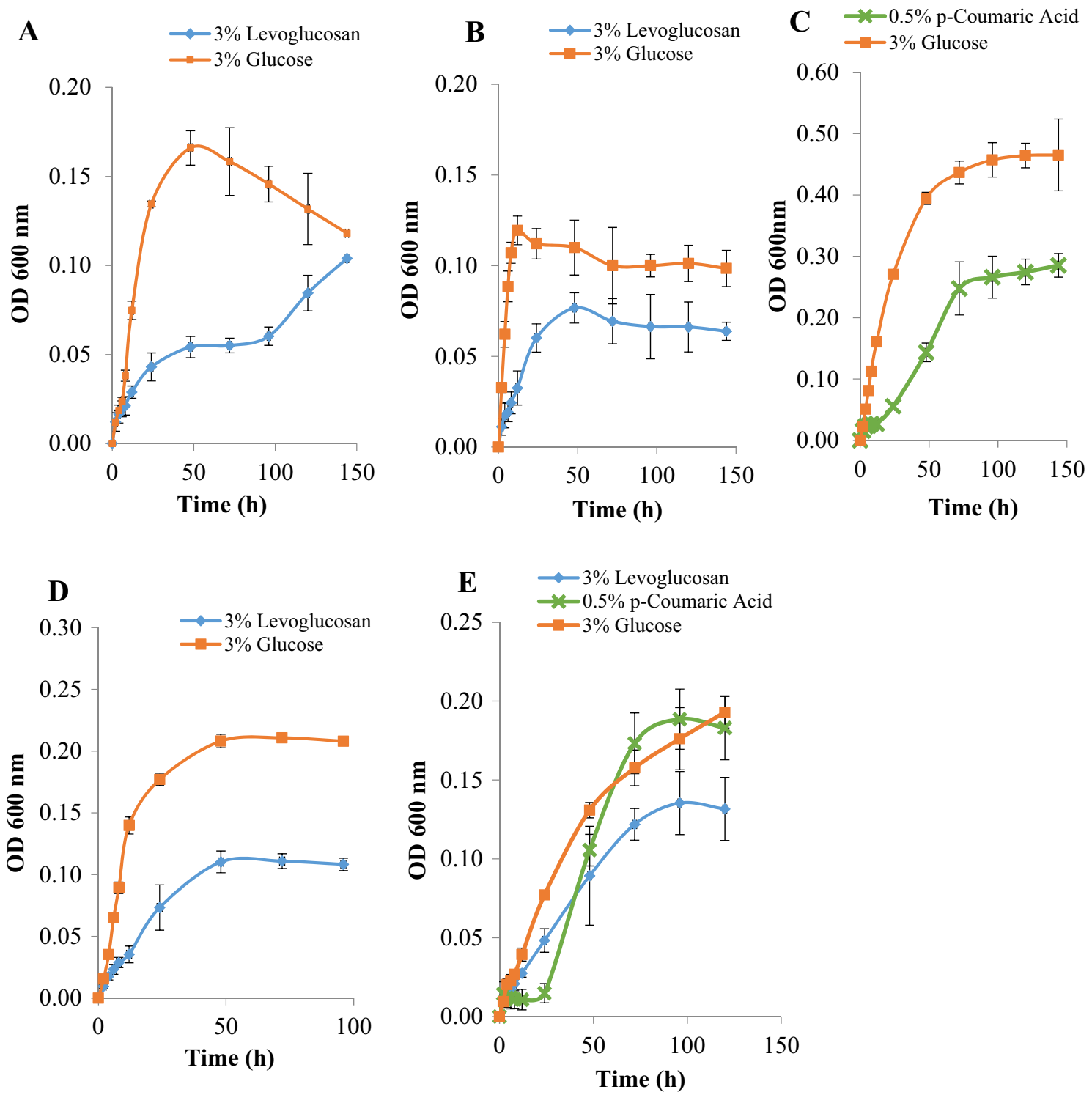

Fig. 3 Growth rates for a Kluyveromyces africanus NCYC 2729, b Kluyveromyces dobzhanskii NCYC 538, c Pichia angusta NCYC 1457, d Pichia etchellsii NCYC 740 and e Pichia membranifaciens
NCYC 101 under control conditions (3\% glucose) and either 3\% levoglucosan (a, b, d, and e) or $0.5 \%$-coumaric acid (c and e). Data are the mean \pm SD of three measurements
Table 3 Utilization of levoglucosan and $p$-coumaric acid by shortlisted yeast strains post anaerobic fermentation (LOD for levoglucosan is $0.003 \mathrm{mg} / \mathrm{L})$

\begin{tabular}{|c|c|c|}
\hline & \multicolumn{2}{|l|}{$\%(\mathrm{w} / \mathrm{v})$} \\
\hline & Levoglucosan & p-Coumaric acid \\
\hline Starting concentration & 3 & 0.5 \\
\hline \multicolumn{3}{|l|}{ End of fermentation concentrations } \\
\hline Pichia membranifaciens NCYC101 & $<0.003 \pm 0.0001$ & $0.5 \pm 0.04$ \\
\hline Pichia etchellsii NCYC740 & $<0.003 \pm 0.0001$ & n.a \\
\hline Pichia angusta NCYC1457 & n.a & $0.5 \pm 0.04$ \\
\hline Kluyveromyces dobzhanskii NCYC538 & $<0.003 \pm 0.0002$ & n.a \\
\hline Kluyveromyces africanus NCYC2729 & $0.63 \pm 0.08$ & n.a \\
\hline
\end{tabular}


Table 4 EIC traces of acetic acid identified in levoglucosan fermentations by shortlisted yeast strains

\begin{tabular}{lll}
\hline Yeast strain & Acetic acid (EIC trace) & $\begin{array}{l}\text { Ethanol } \\
\text { (EIC } \\
\text { trace) }\end{array}$ \\
\hline $\begin{array}{l}\text { Pichia membranifaciens } \\
\text { NCYC101 }\end{array}$ & $6.64 \times 10^{4} \pm 1.40 \times 10^{4}$ & - \\
$\begin{array}{l}\text { Pichia etchellsii NCYC740 } \\
\text { Kluyveromyces dobzhanskii } \\
\text { NCYC538 }\end{array}$ & $5.22 \times 10^{4} \pm 2.31 \times 10^{4}$ & - \\
$\begin{array}{l}\text { Kluyveromyces africanus } \\
\text { NCYC2729 }\end{array}$ & $1.96 \times 10^{4} \pm 8.78 \times 10^{2}$ & - \\
\hline
\end{tabular}

Data are the mean \pm SD of three measurements
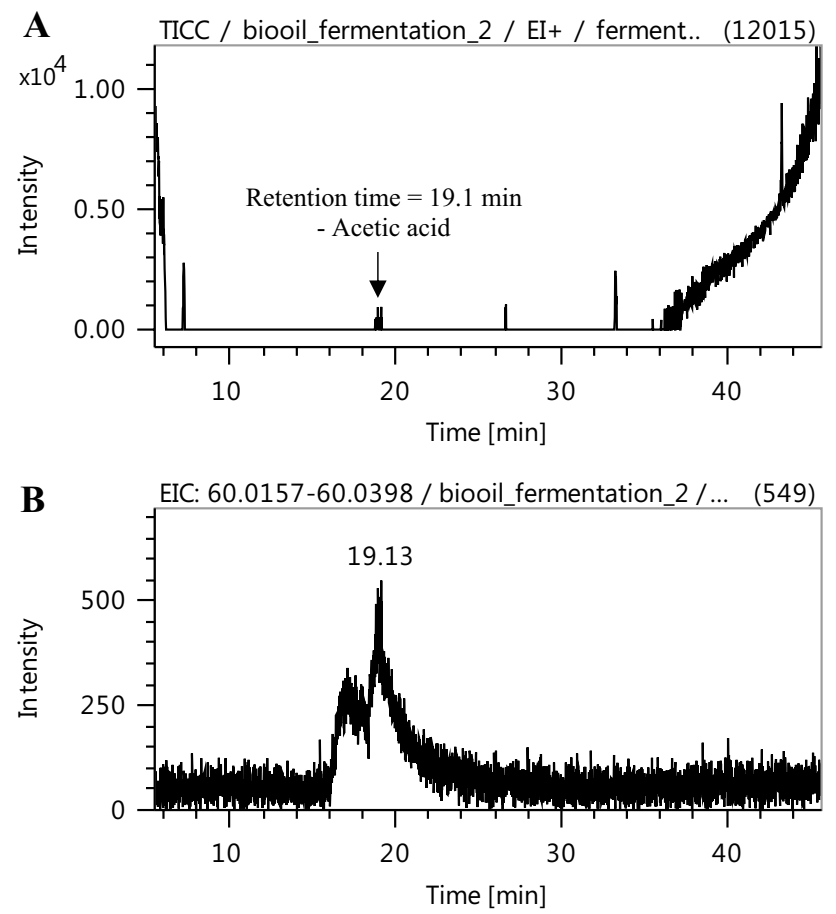

Fig. 4 End of fermentation product screening via GC-MS. Example of chromatograms from the fermentation of $3 \%(w / v)$ levoglucosan (in YPD) by Pichia membranifaciens NCYC101. a Total ion chromatogram (TIC) with labelled acetic acid peak. Unlabelled peaks are either unknowns or derived from the yeast nitrogen peptone (YPD) media. b Extracted ion chromatogram (EIC) traces for acetic acid. The diagnostic ion for acetic acid $(60.025 \pm 0.001 \mathrm{Da})$ is shown from time 16 to $20 \mathrm{~min}$

a trait which is absent in Saccharomyces spp. [36]. Furthermore, $K$. marxianus has been more widely adopted in the biotechnology industry due to its ability to assimilate key sugars (namely lactose and inulin), its thermotolerance, and fast growth rate (as reviewed by [37]). To the authors' knowledge, this is the first report to suggest that Kluyveromyces spp. can metabolise levoglucosan as a sole carbon source and speculate that $K$. africanus NCYC
2729 and $K$. dobzhanskii NCYC 538 may possibly possess levoglucosan utilization mechanisms.

Although the validity of the PM assay was confirmed by growth tests (optical density at $600_{n m}$; Fig. 3a-e), shortlisted strains grew more slowly on levoglucosan and $p$-coumaric acid with the final amount of biomass being lower compared to their growth on glucose. Reasons for the increase in growth for K. africanus NCYC 2729 after $96 \mathrm{~h}$ of incubation on levoglucosan, from around 0.05 to over 0.10 (OD $600_{\mathrm{nm}}$; Fig. 3a) are unclear. However it may be possible that certain genes involved in the metabolism of levoglucosan in the $K$. africanus NCYC 2729 strain may become expressed after $96 \mathrm{~h}$ of incubation.

The conversion of levoglucosan in eukaryotic microorganisms such as yeast and fungi has only thus far been shown to be carried out by the actions of a levoglucosan kinase [21, 35]. Knowledge of the levoglucosan dehydrogenase metabolic pathway is limited, however it catalyses the conversion of levoglucosan in the presence of adenosine triphosphate (ATP) and $\mathrm{Mg}^{2+}$ and the enzymatic reaction involves the simultaneous hydrolysis and phosphorylation of the substrate, yielding D-glucose-6-phosphate and adenosine diphosphate (ADP) [38, 39]. Researchers have successfully been able to isolate and integrate endogenous levoglucosan kinase genes from yeast or fungi into hosts such as E. coli to then investigate levoglucosan consumption rates and product development [8, 21, 22]. However, the levoglucosan kinase enzymes have often displayed a low level binding affinity for the carbon source and not all of the present levoglucosan had been completely up taken by the microbial host [8]. Furthermore, Dai et al. [22] showed that the levoglucosan kinase enzyme present in yeast $L$. starkeyi is relatively active for the conversion of levoglucosan to glucose-6-phosphate. Likewise, the near-complete conversion of levoglucosan into ethanol was obtained by Jarboe et al. [8] after construction of an engineered ethanol producing E.coli (KO11) containing the levoglucosan kinase gene from $L$. starkeyi. Similarly, Linger et al. [24] constructed a Pseudomonas putida (KT2440) strain containing the same levoglucosan kinase gene from $L$. starkeyi but also incorporated an exogenous $\beta$-glucosidase enzyme for the biological hydrolysis of cellobiosan (another major sugar found in pyrolysis bio-oil) into levoglucosan and glucose. These studies have all shown that not only does levoglucosan kinase from the yeast $L$. starkeyi have a high binding affinity for levoglucosan, it is also adequately transferable into multiple hosts. It would be of great interest to investigate whether the yeast strains shortlisted in this study which appeared to be able to metabolize and grow on levoglucosan have a similar, or possibly higher binding affinity for levoglucosan.

Regarding growth on $p$-coumaric acid, $P$. angusta NCYC1457 had a much slower growth rate compared to its growth on the preferable glucose carbon source, however $P$. 
membranifaciens NCYC101 appeared to have a faster growth rate (with a higher biomass $\mathrm{OD}_{600 ;} 0.18 \pm 0.02$ ) on $p$-coumaric acid compared to levoglucosan $\left(\mathrm{OD}_{600 ;} 0.13 \pm 0.04\right)$. Reasons for this are unclear however the identification of a Pichia spp. with abilities to both metabolize and grow on $p$-coumaric acid is novel, warranting further research and assessing their potential as $p$-coumaric acid fermenting yeast strains. Previous studies have revealed that in the presence of $p$-coumaric acid (under aerobic conditions) S. cerevisiae responds by detoxifying $p$-coumaric acid via a series of decarboxylation and oxidation processes into less toxic derivatives, in order for cells to survive and multiply in the present of this compound [40]. The exact metabolic process is unknown and it is also not known if Pichia spp. undergo a similar stress response; yet this may possibly be the case in this present study since there was observed growth and metabolic activity on $p$-coumaric acid.

The ultimate aim of this study was to investigate whether the yeast strains shortlisted from the PM assay and growth test confirmation were able to actually ferment bio-oil compounds levoglucosan and $p$-coumaric acid under anaerobic conditions. Despite the fact that no traceable levels of levoglucosan remained at the end of the fermentation for three out of the four yeast strains under investigation (Table 3), no levels of ethanol were detected. These results are in accordance with reports that levoglucosan utilizers are not able to produce bioethanol [20]. The yeast strain $K$. africanus NCYC2729 was only able to uptake around 79\% levoglucosan, compared to the other strains which had uptake efficiencies of $100 \%$. The levoglucosan kinase which exists in K. africanus NCYC2729 may have a slightly lower affinity for the anhydrosugar, hence its slower uptake compared to $P$. membranifaciens NCYC101, P. etchellsii NCYC740 and $K$. dobzhanskii NCYC538. Surprisingly, all shortlisted yeast candidates detected trace levels of acetic acid (Fig. 4a, b; Table 4) in the fermentations of levoglucosan. It is well documented that during the production of wine, non-Saccharomyces species of yeast produce high levels of undesirable compounds, such as acetoin, ethyl acetate and acetic acid which ultimately affect the quality of wine [41]. Acetic acid, along with other compounds such as terpenoids, fatty acid esters, glycerol and succinic acid are some of the metabolic compounds generated from the growth of nonSaccharomyces spp. and are produced to counteract redox imbalances during the metabolism of sugars [42]. However, the production of acetic acid from the shortlisted yeast strains in this study have not been previously reported and at present it is not definitively feasible to say whether the production of acetic acid is a direct product or a metabolic byproduct; since no traces of ethanol or glycerol were identified. Thermo-tolerant $K$. marxianus IMB strains which were isolated by Banat et al. [43] from a distillery in India have been of particular interest for the production of bioethanol at elevated temperatures above $40{ }^{\circ} \mathrm{C}[44,45]$. These studies revealed that acetic acid (albeit at varying concentrations) was produced alongside bioethanol by these Kluyveromyces spp. from switchgrass via simultaneous saccharification and fermentation at $45{ }^{\circ} \mathrm{C}$. However, the degree of acetic acid production by non-Saccharomyces yeasts may be species specific [46]. It may be a possibility that the shortlisted strains from this study are only able to uptake levoglucosan and not produce any final product; therefore using the carbon source for cell mass production. Alternatively, any carbon end-point product that could have been generated may not be detectable with the techniques used in this study. Utilisation profile studies using larger scale fermentations with the shortlisted yeasts would help to facilitate a determination of the conversion of levoglucosan into acetic acid over time, however this was not feasible during this study due to the cost of obtaining sufficient quantities of levoglucosan. However, it has been suggested that the availability of levoglucosan will increase in the near future, particularly from the development of new and emerging green technologies regarding the processing of biomass, where levoglucosan is a main product $[31,47]$. Nonetheless, the results from this study are still significant since the four shortlisted yeast strains have for the first time been identified as candidates for levoglucosan utilization.

The application of the PM assay as a screening tool has again proved to be useful in identifying candidate strains of yeast for further research on pyrolysis oil compounds. The media which was utilized in the PM assay was synthetic and only contained one compound of interest in order to ensure that that the metabolic output from the yeast strains under investigation were indeed genuine to that particular carbon source. Pyrolysis oil itself is an extremely complex matrix and therefore if pyrolysis oil was used directly in the PM assay it would not be possible to determine contributing factors to the metabolic response. For future studies, it would be desirable to use the pyrolysis oil itself in the PM assay. However, this would require further optimization of the assay to ensure that pyrolysis oil will be compatible with the redox sensitive dyes that are used in the assay to achieve a visible colour change; currently pyrolysis oil masks the visibility of the dye. Additionally, a larger species screen of Pichia and Kluyveromyces yeasts using the PM assay would be a good starting point for further research, as this would help to elucidate whether the traits seen with levoglucosan utilisation are a species wide event or whether they are indeed strain specific.

The present work focussed on strain selection (using the PM assay) and managed to successfully identify novel strains of yeast that can uptake levoglucosan under anaerobic fermentation conditions. Although no traces of ethanol were detected in the fermentations performed with these shortlisted strains, trace amounts of acetic acid were identified. 
There is still much more research required with these candidate yeast strains in order to identify key pathways and uptake mechanisms that are behind the levoglucosan utilization, which are clearly not present in all species belonging to the same genus. Discovering new genes from wildtype strains of yeast that can be transformed into efficient microbial vectors or 'workhorses' such as E. coli to ferment unique pyrolytic carbon sources will need to be successfully accomplished to make the biological upgrading of pyrolysis bio-oil become a reality.

Acknowledgements ETK and JPR would like to sincerely thank the International Centre for Brewing Science for access to the yeast culture collection and use of the Biolog-Omnilog phenotypic microarray.

\section{Compliance with Ethical Standards}

Conflict of interest The authors declare that they have no conflict of interest.

Open Access This article is distributed under the terms of the Creative Commons Attribution 4.0 International License (http://creativeco mmons.org/licenses/by/4.0/), which permits unrestricted use, distribution, and reproduction in any medium, provided you give appropriate credit to the original author(s) and the source, provide a link to the Creative Commons license, and indicate if changes were made.

\section{References}

1. Arnold, S., Moss, K., Henkel, M., Hausmann, R.: Biotechnological perspectives of pyrolysis oil for a bio-based economy. Trends Biotechnol. 35, 925-936 (2017)

2. Bridgwater, A., Meier, D., Radlein, D.: An overview of fast pyrolysis of biomass. Org. Geochem. 30, 1479-1493 (1999)

3. Oasmaa, A., Czernik, S.: Fuel oil quality of biomass pyrolysis oils state of the art for the end users. Energy Fuels 13, 914-921 (1999)

4. Vitasari, C.R., Meindersma, G., De Haan, A.B.: Water extraction of pyrolysis oil: the first step for the recovery of renewable chemicals. Bioresour. Technol. 102, 7204-7210 (2011)

5. Brown, R.C.: Hybrid thermochemical/biological processing. Appl. Biochem. Biotechnol. 137, 947-956 (2007)

6. Brown, R.C.: Biomass refineries based on hybrid thermochemicalbiological processing - an overview. In: Kamm, B., Gruber, P.R., Kamm, M. (eds.) Biorefineries-Industrial Processes and Products: Status Quo and Future Directions, vol. 1, pp. 227-252. Wiley, Weinheim (2006)

7. Shen, Y., Jarboe, L., Brown, R., Wen, Z.: A thermochemical-biochemical hybrid processing of lignocellulosic biomass for producing fuels and chemicals. Biotechnol. Adv. 33, 1799-1813 (2015)

8. Jarboe, L.R., Wen, Z., Choi, D., Brown, R.C.: Hybrid thermochemical processing: fermentation of pyrolysis-derived bio-oil. Appl. Biochem. Biotechnol. 91, 1519 (2011)

9. Yu, Z., Zhang, H.: Ethanol fermentation of acid-hydrolyzed cellulosic pyrolysate with Saccharomyces cerevisiae. Bioresour. Technol. 90, 95-100 (2003)

10. Yu, Z., Zhang, H.: Pretreatments of cellulose pyrolysate for ethanol production by Saccharomyces cerevisiae, Pichia sp. YZ-1 and Zymomonas mobilis. Biomass Bioenergy 24, 257-262 (2003)
11. Bennett, N.M., Helle, S.S., Duff, S.J.: Extraction and hydrolysis of levoglucosan from pyrolysis oil. Bioresour. Technol. 100, 6059-6063 (2009)

12. Lian, J., Chen, S., Zhou, S., Wang, Z., O'Fallon, J., Li, C.-Z., Garcia-Perez, M.: Separation, hydrolysis and fermentation of pyrolytic sugars to produce ethanol and lipids. Bioresour. Technol. 101, 9688-9699 (2010)

13. Luque, L., Westerhof, R., Van Rossum, G., Oudenhoven, S., Kersten, S., Berruti, F., Rehmann, L.: Pyrolysis based bio-refinery for the production of bioethanol from demineralized ligno-cellulosic biomass. Bioresour. Technol. 161, 20-28 (2014)

14. Helle, S., Bennett, N.M., Lau, K., Matsui, J.H., Duff, S.J.: A kinetic model for production of glucose by hydrolysis of levoglucosan and cellobiosan from pyrolysis oil. Carbohydr. Res. 342, 2365-2370 (2007)

15. Kitamura, Y., Abe, Y., Yasui, T.: Metabolism of levoglucosan (1, 6 -anhydro- $\alpha$-D-glucopyranose) in microorganisms. Agric. Biol. Chem. 55, 515-521 (1991)

16. Nakagawa, M., Sakai, Y., Yasui, T.: Itaconic acid fermentation of lavoglucosan. J. Ferment. Technol. 62, 201-203 (1984)

17. Nakahara, K., Kitamura, Y., Yamagishi, Y., Shoun, H., Yasui, T.: Levoglucosan dehydrogenase involved in the assimilation of levoglucosan in Arthrobacter sp. I-552. Biosci. Biotechnol. Biochem. 58, 2193-2196 (1994)

18. Zhuang, X., Zhang, H.: Identification, characterization of levoglucosan kinase, and cloning and expression of levoglucosan kinase cDNA from Aspergillus niger CBX-209 in Escherichia coli. Protein Expr. Purif. 26, 71-81 (2002)

19. Xie, H., Zhuang, X., Bai, Z., Qi, H., Zhang, H.: Isolation of levoglucosan-assimilating microorganisms from soil and an investigation of their levoglucosan kinases. World J. Microbiol. Biotechnol. 22, 887-892 (2006)

20. Islam, Z.U., Zhisheng, Y., Dongdong, C., Hongxun, Z.: Microbial conversion of pyrolytic products to biofuels: a novel and sustainable approach toward second-generation biofuels. J. Ind. Microbiol. Biotechnol. 42, 1557-1579 (2015)

21. Layton, D.S., Ajjarapu, A., Choi, D.W., Jarboe, L.R.: Engineering ethanologenic Escherichia coli for levoglucosan utilization. Bioresour. Technol. 102, 8318-8322 (2011)

22. Dai, J., Yu, Z., He, Y., Zhang, L., Bai, Z., Dong, Z., Du, Y., Zhang, H.: Cloning of a novel levoglucosan kinase gene from Lipomyces starkeyi and its expression in Escherichia coli. World J. Microbiol. Biotechnol. 25, 1589 (2009)

23. Xiong, X., Lian, J., Yu, X., Garcia-Perez, M., Chen, S.: Engineering levoglucosan metabolic pathway in Rhodococcus jostii RHA1 for lipid production. J. Ind. Microbiol. Biotechnol. 43, 1551-1560 (2016)

24. Linger, J.G., Hobdey, S.E., Franden, M.A., Fulk, E.M., Beckham, G.T.: Conversion of levoglucosan and cellobiosan by Pseudomonas putida KT2440, Metab. Eng. Commun. 3, 24-29 (2016)

25. Greetham, D., Wimalasena, T.T., Leung, K., Marvin, M.E., Chandelia, Y., Hart, A.J., Phister, T.G., Tucker, G.A., Louis, E.J., Smart, K.A.: The genetic basis of variation in clean lineages of Saccharomyces cerevisiae in response to stresses encountered during bioethanol fermentations. PLoS ONE 9, e103233 (2014)

26. Greetham, D., Wimalasena, T., Kerruish, D., Brindley, S., Ibbett, R., Linforth, R., Tucker, G., Phister, T., Smart, K.: Development of a phenotypic assay for characterisation of ethanologenic yeast strain sensitivity to inhibitors released from lignocellulosic feedstocks. J. Ind. Microbiol. Biotechnol. 41, 931-945 (2014)

27. Wimalasena, T.T., Greetham, D., Marvin, M.E., Liti, G., Chandelia, Y., Hart, A., Louis, E.J., Phister, T.G., Tucker, G.A., Smart, K.A.: Phenotypic characterisation of Saccharomyces spp. yeast for tolerance to stresses encountered during fermentation of lignocellulosic residues to produce bioethanol. Microb. Cell Fact. 13, 47 (2014) 
28. Wilkinson, S., Greetham, D., Tucker, G.A.: Evaluation of different lignocellulosic biomass pretreatments by phenotypic microarraybased metabolic analysis of fermenting yeast. Biofuel Res. J. 3, 357-365 (2016)

29. Kostas, E.T., White, D.A., Du, C., Cook, D.J.: Selection of yeast strains for bioethanol production from UK seaweeds. J. Appl. Phycol. 28, 1427-1441 (2016)

30. Robinson, J., Dodds, C., Stavrinides, A., Kingman, S., Katrib, J., Wu, Z., Medrano, J., Overend, R.: Microwave pyrolysis of biomass: control of process parameters for high pyrolysis oil yields and enhanced oil quality. Energy Fuels 29, 1701-1709 (2015)

31. Shepherd, B.J., Ryan, J., Adam, M., Vallejo, D.B., Castaño, P., Kostas, E.T., Robinson, J.P.: Microwave pyrolysis of biomass within a liquid medium. J. Anal. Appl. Pyrolysis 134, 381-388 (2018)

32. Zhisheng, Y., Jiying, N., Ling, Z., Hongxun, Z.: Microbial investigation on assimilation and ethanol fermentation of levoglucosan from cellulosic pyrolysis products. Chin. Agric. Sci. Bull. 6, 070 (2011)

33. Lian, J., Garcia-Perez, M., Chen, S.: Fermentation of levoglucosan with oleaginous yeasts for lipid production. Bioresour. Technol. 133, 183-189 (2013)

34. Ning, J., Yu, Z., Xie, H., Zhang, H., Zhuang, G., Bai, Z., Yang, S., Jiang, Y.: Purification and characterization of levoglucosan kinase from Lipomyces starkeyi YZ-215. World J. Microbiol. Biotechnol. 24, 15-22 (2008)

35. Prosen, E.M., Radlein, D., Piskorz, J., Scott, D.S., Legge, R.L.: Microbial utilization of levoglucosan in wood pyrolysate as a carbon and energy source. Biotechnol. Bioeng. 42, 538-541 (1993)

36. Lane, M.M., Morrissey, J.P.: Kluyveromyces marxianus: a yeast emerging from its sister's shadow. Fungal Biol. Rev. 24, 17-26 (2010)

37. Fonseca, G.G., Heinzle, E., Wittmann, C., Gombert, A.K.: The yeast Kluyveromyces marxianus and its biotechnological potential. Appl. Microbiol. Biotechnol. 79, 339-354 (2008)

38. Rother, C., Gutmann, A., Gudiminchi, R., Weber, H., Lepak, A., Nidetzky, B.: Biochemical characterization and mechanistic analysis of the levoglucosan kinase from Lipomyces starkeyi. ChemBioChem 19, 596-603 (2018)

39. Bacik, J.P., Jarboe, L.R.: Bioconversion of anhydrosugars: emerging concepts and strategies. IUBMB Life 68, 700-708 (2016)
40. Adeboye, P.T., Bettiga, M., Aldaeus, F., Larsson, P.T., Olsson, L.: Catabolism of coniferyl aldehyde, ferulic acid and p-coumaric acid by Saccharomyces cerevisiae yields less toxic products. Microb. Cell Fact. 14, 149 (2015)

41. Comitini, F., Gobbi, M., Domizio, P., Romani, C., Lencioni, L., Mannazzu, I., Ciani, M.: Selected non-Saccharomyces wine yeasts in controlled multistarter fermentations with Saccharomyces cerevisiae. Food Microbiol 28, 873-882 (2011)

42. García, M., Esteve-Zarzoso, B., Arroyo, T.: Non-saccharomyces yeasts: biotechnological role for wine production. In: Grape and Wine Biotechnology. InTech (2016). https://doi. org/10.5772/64957

43. Banat, I.M., Nigam, P., Marchant, R.: Isolation of thermotolerant, fermentative yeasts growing at $52{ }^{\circ} \mathrm{C}$ and producing ethanol at $45^{\circ} \mathrm{C}$ and $50{ }^{\circ} \mathrm{C}$. World J. Microbiol. Biotechnol. 8, 259-263 (1992)

44. Suryawati, L., Wilkins, M.R., Bellmer, D.D., Huhnke, R.L., Maness, N.O., Banat, I.M.: Simultaneous saccharification and fermentation of Kanlow switchgrass pretreated by hydrothermolysis using Kluyveromyces marxianus IMB4. Biotechnol. Bioeng. 101, 894-902 (2008)

45. Faga, B.A., Wilkins, M.R., Banat, I.M.: Ethanol production through simultaneous saccharification and fermentation of switchgrass using Saccharomyces cerevisiae D 5 A and thermotolerant Kluyveromyces marxianus IMB strains. Bioresour. Technol. 101, 2273-2279 (2010)

46. Erasmus, D.J., Cliff, M., van Vuuren, H.J.: Impact of yeast strain on the production of acetic acid, glycerol, and the sensory attributes of icewine. Am. J. Enol. Vitic. 55, 371-378 (2004)

47. Sagedashi, M., Miyasaka, N., Shishido, H., Sokada, A.: Superheated steam pyrolysis of biomas elemental components and Sugi (Japanese cedar) for fuels and chemicals. Bioresour. Technol. 97, 1272-1283 (2006)

Publisher's Note Springer Nature remains neutral with regard to jurisdictional claims in published maps and institutional affiliations.

\section{Affiliations}

\section{Emily T. Kostas ${ }^{1,2} \mathbb{D} \cdot$ Mick Cooper $^{3} \cdot$ Benjamin J. Shepherd $^{1} \cdot$ John P. Robinson $^{1}$}

Emily T. Kostas

e.kostas@ucl.ac.uk

1 Microwave Process Engineering Research Group, Faculty of Engineering, The University of Nottingham, Nottingham NG7 5RD, UK

2 Present Address: Department of Biochemical Engineering, The Advanced Centre for Biochemical Engineering, Bernard
Katz Building, University College London, Gower Street, London WC1H 6BT, UK

3 School of Chemistry, The University of Nottingham, Nottingham NG7 5RD, UK 